\title{
THE EXAMINATION OF TEETH IN GROUP MEDICINE
}

\author{
By BOYD S. GARDNER, D.D.S., Rochester, Minnesota
}

(Read before the Southern Minnesota Medical.Association, Mankato, December, 1921)

$\mathrm{T}$

HE literature on dental subjects for the last few years does not give credit to the value of full mouth roentgenograms and the necessity of such examination apparently is not entirely appreciated either by the medical or the dental professions. Certain members of both professions still consider it quite ridiculous to examine, roentgenographically, the mouth of a patient wearing a full upper or lower denture. However, Eusterman's findings, in a study of roentgenograms of the mouths of 290 patients wearing either full or partial dentures, have demonstrated the importance of this phase of our work. Obviously a full mouth examination not only is necessary, but the patient's due, if he is suffering from a systemic condition and a focus of infection is suspected. Besides this group of patients are those who are undergoing thorough examinations from the standpoint of prevention of disease.

In diagnosing affections of the teeth, the existing pathologic condition should be visualized as it is when the teeth are removed surgically. The open view operation has made this possible since the exact condition may be seen, while with the older methods it is necessary to work more or less in the dark. For this reason it is important that the dental diagnostician should have surgical training.

If complete extraction is necessary and a full denture required, especially in elderly persons, it is well to explain carefully the probable results. Many patients are shocked by the change and regret having had the extraction. While the elimination of dental infection is of primary consideration, nevertheless the dental surgeon should keep in mind that the patients are to have dentures and that cooperation with the prosthodontist should begin before operation. In many instances pre-cperative models and facial measurements are advantageous.

It has been proved beyond doubt that a casual glance into the mouth with the idea of noting suspicious teeth, such as those supporting bridges and crowns, or holding large fillings, and so forth, is worthless. The deciding question is whether or not the clinician actually wishes to know if the teeth with their investing tissues are to be considered possible factors in the condition of the patient. This conclusion can only be reached after the examination of thousands of patients by full mouth roentgenograms. Residual roots, granulomas, and impacted teeth are often found under plates that have been worn for many years, and their removal has relieved many patients suffering from systemic disorders, particularly iritis, and one type of arthritis, neuritis, and so forth.

The roentgen-ray evidence should be

Jour. A. D. A., A ugust, 1922 
carefully considered while the patient is under observation. The many advantages in checking up the clinical and roentgen-ray evidence at the same time cannot be overestimated. The full mouth roentgenogram makes it possible to compare densities, which is just as necessary in dental work as in orthopedic work, when, for example, an abnormal condition of the right knee can best be demonstrated roentgenographically by a comparison with a normal left knee. Likewise in dentistry, the lower first molar may appear to be abnormal, but on comparison with the opposite first molar, one may be surprised to see that the condition is normal.

During the past year, approximately 28,000 patients were examined in the dental section of the Mayo Clinic. A review of the histories of these patients disclose ample evidence that the usual method of extraction is far from adequate. Residual roots and granulomas, as well as impacted and unerupted teeth were not infrequently found in supposed edentulous areas. Thirty per cent of these patients had roots, 4 per cent had impacted or unerupted teeth, and 3.5 per cent had residual granulomas. With the pen view operation of extraction which is now known as alveolotomy, it is practically impossible to overlook such conditions; this is proved by postoperative roentgenograms made routinely. The literature abounds in criticism of this method, yet when it is carried out with proper restriction the results are most satisfactory.

Physicians should encourage the routine examination of teeth except in hopeless or emergency cases. Time is an important factor in emergency cases; however, the dental examination can be postponed until during convalescence after operation. The condition for which the patient is operated need not necessarily be due to mouth infection to warrant the dental examination, but the elimination of such infection is of material aid in the after care of the patient, and in the prognosis.

Oral infection cannot be eliminated by extraction alone. Treatment for pyorrhea and thorough prophylaxis are both often necessary. Great care should be exercised with regard to the number of teeth extracted at one time, and the length of time between operations. This is also true of prophjlactic treatments. There should be a limited amount of scaling at the first appointment and it should not be repeated for several days.

The profession of dentistry should be discouraged from making promises to patients as to the possible result following the extraction of teeth with evidence of infection. They need the co-operation of the physician and are only responsible for the field in which they work. An intelligent report with regard to the dental findings is desired from the dentist by the physician who should take the responsibility of advising and encouraging the patient as to the outcome of the dental treatment.

The dentist in making his report divides the teeth into two groups: those which should be removed for local reasons, and those which should be removed when systemic conditions warrant the procedure. Therefore, the removal of teeth in patients in the first group is a question for the dentist to decide, providing the patient's physical condition is such that they can stand the operation, and of those of the second for the physician. Teeth with marked evidence of infection at the roots, certain types of impactions and teeth affected by pyorrhea to such an extent that treatment is of no avail, compose the first group. In the second group are listed pulpless teeth that are negative to the Roentgen-ray, and their extraction is 
advised only when the systemic condition is traced to a focus. Thus many patients are allowed to retain pulpless teeth, which however should always be considered questionable. Therefore, to say that all pulpless teeth should be extracted is doubtless too radical at the present time.

\section{Conclusions}

1. It is necessary that the dental report shall be based on the Roentgen-ray, making use of full mouth pictures, and by clinical evidence.

2. Co-operation between the medical and dental professions is necessary in order that concusions may be reached with regard to the teeth which should be sacrificed.

3. The dentist should be discouraged from making promises with regard to the outcome of extractions.

4. The extraction of teeth by simple pulling is not adequate or justifiable.

5. The results from the extractions of teeth by the method known as alveolotomy should be encouraged with proper restrictions.

\section{BIBLIOGRAPHY}

Eusterman, M. F. "Roentgenographic Findings in Two Hundred and Ninety Partially Edentulous or Edentulous Mouths," Dental Cosmos, I.XIII (1921). 901-3.

\section{Discussion}

Thomas B. Hartzell, Minneapolis: Partial examinations are only misleading, and I would like to urge that in all cases, after removal of diseased teeth, checking up of the work by a radiograph is necessary, and I believe most men will agree in a short time that is important and should be done. In addition to a complete X-ray examination, emphasis should be placed on the fact that in co-operating with medical men the dentist should have a complete diagnosis of his patient. If a dentist is advised to extract teeth by the physician without knowing what the physical condition of the patient is he must rely on his own judgment and his judgment cannot be complete without a good foundation laid by himself or by the physician. If the physician does not hand him this advice with the patient, he is under the necessity of making the diagnosis himself. I have employed a technician for some years at considerable expense, and I operate on no case without a proper and complete examination.

In regard to removing bone from the labial surfaces of the teeth in making extractions, I know that it is done under proper restrictions. As I understand it, the idea now is that wo will not sacrifice the alveolar process of the jaws any more than is absolutely necessary. I am absolutely in accord with that proposition. There has been a phrase among dental operators for some two or three years back to peel back the periosteum, to traumatize the tissues and remove so much of the alveolar process that many of these patients have been unable to wear artificial dentures. Any one who is robbed of his masticating organs and cannot wear an artificial denture is in an unhappy, uncomfortable position. Dr. Gardner cautions us, and it pleases me, because it is a step in the right direction. We do not want to sacrifice the alveolar process of jaws of patients who must subsequently wear artificial dentures. 